\title{
Estudio ecológico de los bosques homogéneos en el distrito de Molinopampa, Región Amazonas
}

\section{Ecological study of homogeneous forests in the district of Molinopampa, Amazonas Region}

Aguinaldo García-Pérez ${ }^{1}$, Karol Brighton Rubio Rojas ${ }^{1}$, Jegnes Benjamin Meléndez Mori ${ }^{1}$, Fernando Corroto ${ }^{1}$, Jesús Rascón ${ }^{1}$, Manuel Oliva

\section{RESUMEN}

En el presente trabajo se caracterizó el bosque homogéneo de palmeras en el distrito de Molinopampa, ya que esta área es representativa de este tipo de bosques, los cuales se ven afectados por actividades agropecuarias. En se sentido, se cuantificaron e identificaron las especies de palmeras de este bosque de palmera, así como la distribución en el área de estudio, encontrándose que, en un área de aproximadamente 5000 hectáreas solo se presentan cuatro especies del genero Ceroxylon, las cuales son Ceroxylon peruvianum, Ceroxylon quidiuense, Ceroxylon vogelianum y Ceroxylon parvifrons. De todas, la que tiene mejor distribución y frecuencia, al encontrarse en todos los puntos de muestro, fue C. quindiuense, misma que representa el $73 \%$ del total de individuos muestreados. Las demás especies no son muy representativas y solo se han encontraron en un punto de muestreo. Esta baja diversidad de especies encontrada se debe a la interacción del bosque de palmeras con actividades antrópicas como la agricultura y la ganadería.

Palabras claves: Ceroxylon, palmeras, diversidad, índice de Shannon-Wiener.

\begin{abstract}
In the present work, the homogeneous forest of palm trees in the district of Molinopampa was characterized, since this area is representative of this type of forests, which are affected by agricultural activities. In this sense, the palm tree species of this palm forest were quantified and identified, as well as the distribution in the study area, finding that, in an area of approximately 5000 hectares, only four species of the genus Ceroxylon are present, which are Ceroxylon peruvianum, Ceroxylon quidiuense, Ceroxylon vogelianum and Ceroxylon parvifrons. Of all, the one that has better distribution and frequency, when being in all the points of sample, was $C$. quindiuense, same that represents $73 \%$ of the total of sampled individuals. The other species are not very representative and have only been found at one sampling point. This low diversity of species found is due to the interaction of the palm forest with anthropogenic activities such as agriculture and livestock.
\end{abstract}

Keywords: Ceroxylon, palm trees, diversity, Shannon-Wiener index.

\footnotetext{
Universidad Nacional Toribio Rodríguez de Mendoza de Amazonas (UNTRM-A), Instituto de Investigación para el Desarrollo Sustentable de Ceja de Selva, Calle Higos Urco N 342-350-356, Calle Universitaria N ${ }^{\circ} 304$, Chachapoyas, Perú

"Autor de correspondencia. E-mail: soliva@indes-ces.edu.pe
} 


\section{INTRODUCCIÓN}

La familia Arecaceae tiene 182 géneros con 2600 especies, la mayoría prosperan en climas tropicales y subtropicales (Baker y Dransfield, 2016). Sin embargo, existen bosques de palmeras en climas templados fríos. En ese sentido en el año 2008, se reporta el primer registro de la especie Ceroxylon peruvianum en los Andes del Norte de Perú. Además, se encontró miles de individuos de Ceroxylon quindiuense, otra especie de palmera en la localidad de Ocol, cerca de Chachapoyas, en el departamento de Amazonas (Galeano et al., 2008), confirmándose la importancia de estas especies del genero Ceroxylon para los bosques de Amazonas. En 2011 se amplío la distribución de Ceroxylon quindiunse para la región de Cajamarca en Perú (Santa-Cruz, 2011).

De acuerdo a Manturano (2007), los estudios ecológicos que nos suministran información para el manejo de los bosques en Perú, no alcanzan el 20\% de los principales ecosistemas. Mientras que en el caso de los bosques donde las asociaciones dominantes son las palmeras, siendo ecosistemas de gran importancia, los estudios son casi nulos dado el grado de ocupación que tiene la agricultura. Es por ello, que conocer su ecología y valor económico es de vital importancia, sin embargo, los bosques de palmeras han sido poco estudiados, puesto que las especies arbóreas que proporcionan madera han sido las más deforestadas (Hubbell y Foster 1986; Clark et al., 2017; Clark et al., 2018). Actualmente, el avance acelerado de la destrucción de los bosques, especialmente los que contienen buen número de palmeras son óptimos para la agricultura y el establecimiento de pastos, gracias a las características edáficas de estos bosques, sin dejar de lado la extracción de madera y de plantas medicinales (Mas et al., 2017; Flores et al., 2017).

Tener conocimiento acerca de las especies con las que cuenta los bosques y su comportamiento estructural, genera información útil para iniciar programas de protección y mitigación de problemas asociados a estos bosques. En el del distrito de Molinopampa casi la totalidad de la población se dedica a la actividad ganadera, convirtiéndose en su principal fuente de ingresos económicos lo que provoca una deforestación de los bosques naturales de palmeras de Ocol, Pumahermana Santa Rosa y San José, anexos que comprenden uno de los bosques de palmeras más importantes de Perú (Oliva et al., 2017). Es por ello, que conocer la estructura horizontal de las palmeras en el distrito de Molinopampa, extenderá el conocimiento acerca de las problemáticas que puedan estar ocurriendo en referencia a estos bosques, al determinar las variables relacionadas con la diversidad y frecuencia de palmeras en bosques asociados a pasturas y en bosques primarios, además de calcular su abundancia.

\section{MATERIALES Y MÉTODOS}

El presente estudio se realizó en el distrito de Molinopampa, provincia Chachapoyas región Amazonas, limitando por el norte con el distrito de Quinjalca y el distrito de Granada; por el sureste con la provincia de Rodríguez de Mendoza; por el suroeste con el distrito de Cheto; y por el oeste con el distrito de San Francisco de Daguas y el distrito de Sonche. Ubicándose entre las coordenadas de $6^{\circ} 19^{\prime} 0.53^{\prime \prime}$ de latitud sur y $77^{\circ} 29^{\prime}$ 39.52"de longitud oeste a 2401 m.s.n.m. (Figura 1).

Realizando un recorrido por el distrito se delimitaron las áreas de bosque de palmeras de Ocol, Pumahermana, San José y Santa Rosa, para generar un mapa de la extensión de estos bosques se utilizó un GPS Garmin 550 Oregón procesando los datos tomados con el Software ArcGis 10.4 y MapSource.

El conteo de palmeras, se realizó estableciendo 16 transectos con las dimensiones de 5 x 500 metros cada uno. Considerando los usos de los bosques se decidió distribuir estos transectos en dos ubicaciones, ocho bosques de palmeras asociados a pasturas y ocho en bosques de palmeras primarios, además se tuvo en consideración su ubicación para que se encuentre representada toda el área de estudio, es decir los anexos de San José, Pumahermana, Ocol y Santa Rosa. Los transectos fueron codificados y se orientaron teniendo en cuenta las condiciones geográficas del lugar, para mantener la proyección horizontal de los 

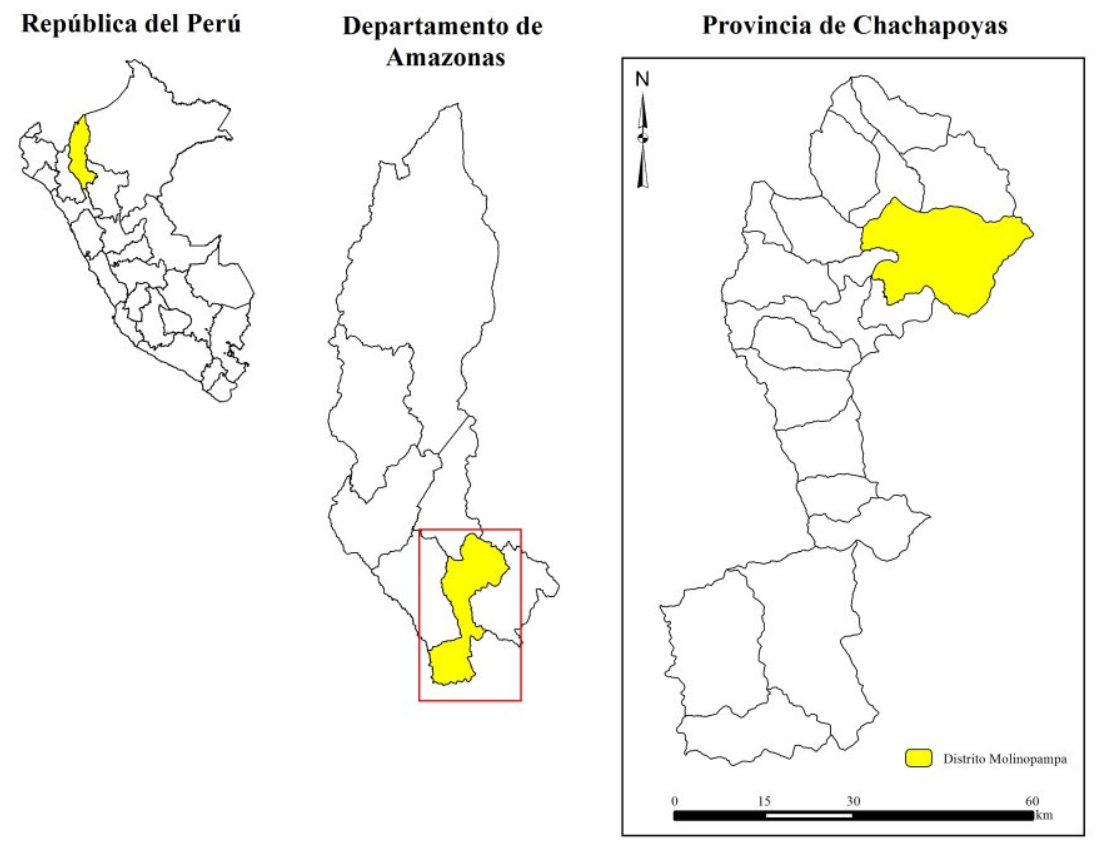

Figura 1. Ubicación geográfica de la zona de estudio.

mismos se colocaron estacas a intervalos de $5 \mathrm{~m}$. Para evaluar y cuantificar la riqueza, la diversidad y la abundancia de especies de palmeras del género Ceroxylon, se recolectaron en cada transecto hojas, tallos, inflorescencias y frutos para la identificación de especies. Además se tomaron muestras de suelo para la caracterización edáfica. Estas muestras se tomaron de a una profundidad de $30 \mathrm{~cm}$, tres submuestras por cada transecto se tomaron al inicio del mismo $(0 \mathrm{~m})$, en la parte media $(250 \mathrm{~m})$ y al final $(500 \mathrm{~m})$, cada muestra fue codificada y georreferenciada.

La identificación y cuantificación de palmeras se realizó, tomando muestras de todos los individuos ubicados dentro del transecto, los cuales fueron clasificados en cuatro categorías: plántulas que son individuos todavía enlazados a la semilla o con hojas enteras; juveniles que portan hojas divididas, pero que un no han iniciado su reproducción; los su adultos que fueron del mismo tamaño que los adultos, pero $\sin$ signos de reproducción; y los adultos que han alcanzado la etapa reproductiva. Todo ello, para lo confección de un herbario que permita la identificación de las especies a través de recolectar especímenes botánicos de plántulas, juveniles, su adultas y adultas para las identificaciones. Cada muestra recolectada fue codificada y se tomaron las coordenadas y datos de la palme- ra de la cual se obtuvo.

En el Laboratorio de Investigación de Suelos y Aguas (LABISAG) de la Universidad Nacional Toribio Rodríguez de Mendoza (UNTRM) se realizó la identificación de especies con las claves taxonómicas de (Galeano et al., 2008; Henderson et al., 1995); además, del análisis de los suelos determinando su clase textural mediante la técnica del Hidrómetro de Bouyoucos, $\mathrm{pH}$ utilizando un potenciómetro, y la conductividad eléctrica mediante el uso de un conductímetro. Para determinar parámetros estructurales de los bosques de palmeras se midieron la abundancia, frecuencia y diversidad de especies.

\section{RESULTADOS Y DISCUSIÓN}

La registraron 5760,8 hectáreas de extensión de bosque de palmeras en el distrito de Molinopampa ubicados entre los anexos de Ocol, Pumahermana, San José y Santa Rosa (Figura 2).

En el área identificada se reportan cuatro especies de palmeras, pertenecientes al Género Ceroxylon, dentro de la familia de las Aracaceas, estas fueron Ceroxylon parvifrons (Engel) H. Wendl., Ceroxylon peruvianum Galeano, Sanín y K. Mejía, Ceroxylon quindiuense (H. Karst.) H. Wendl., Ceroxylon vogelianum (Engel) H. Wendl., distribuidas en los anexos estudiados. Se 


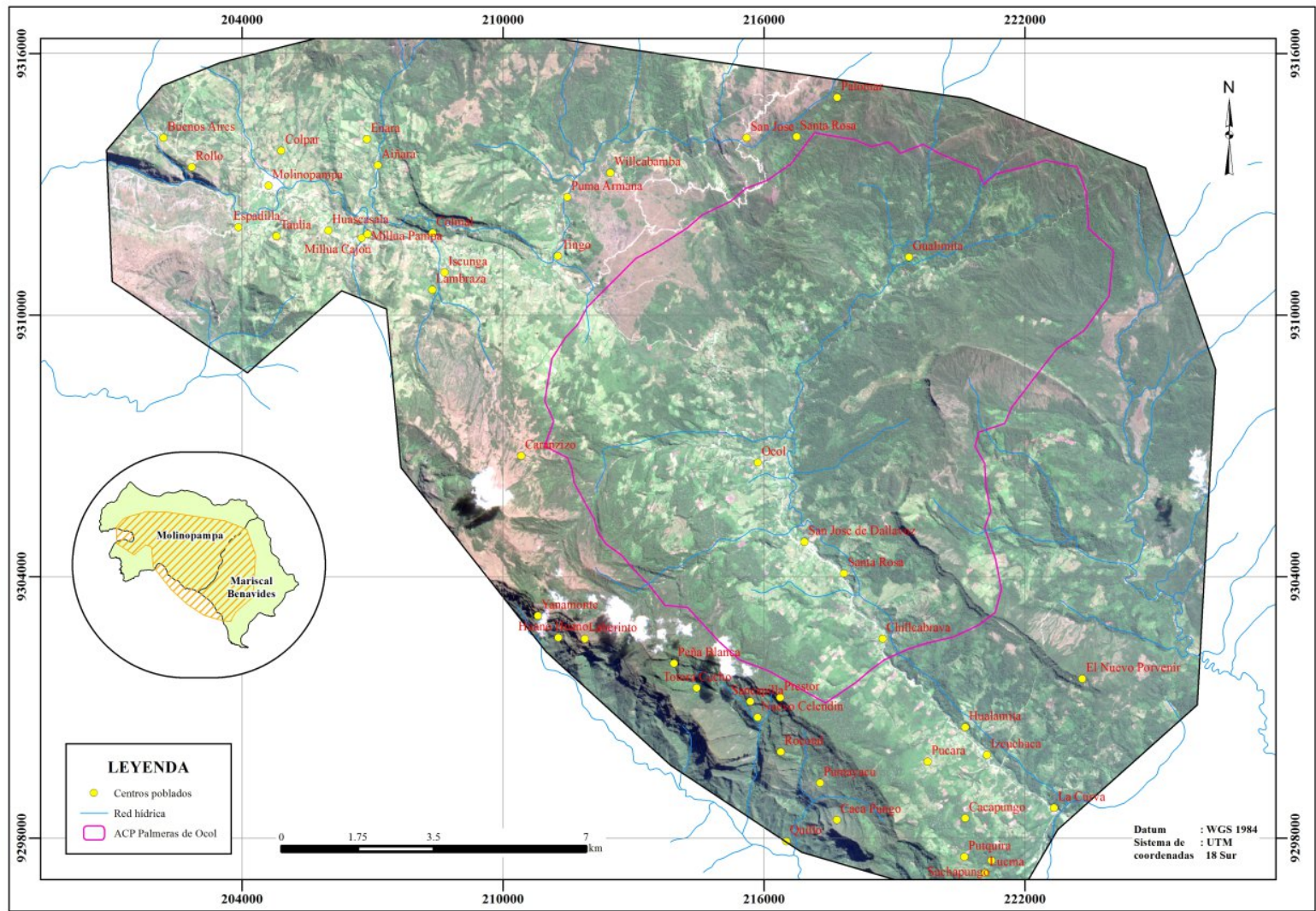

Figura 2. Mapa de limitación del área de la zona de investigación.

encontraron poblaciones de palmeras distribuidas aleatoriamente con algunas repercusiones por parte de las actividades agropecuarias de los pobladores, interviniendo parte del bosque primario. Las 4 especies del género Ceroxylon encontrados en nuestro estudio de las comunidades de palmeras del distrito de Molinopampa representan alrededor de menos de un tercio de las especies de los géneros reportados para Perú (Kahn y Moussa 1994).

El distrito de Molinopampa constituye un hábitat propicio para el desarrollo del género Ceroxylon, ya que presenta características especiales de bosque húmedo montano, y altitudes sobre las que logra prosperar dicha especie, distribuidas entre 1500- 3500 m.s.n.m. Pero sin embargo, se identificaron 4 de las 11 especies descritas por (Henderson et al., 1995). Esto, se debe posiblemente a la fragmentación del hábitat, que genera un efecto negativo sobre la dispersión de las especies, en relación con una reducción de la abundancia y diversidad de los dispersores (Wright y Duber, 2001), lo que se vio reflejado también con el índice de Shannon Wiener, que indicó una baja diversidad de especies del género Ceroxylon en el área de estudio. Hay lugares más diversos como la región de Madre de Dios en Perú, los estudios en el valle del río Ucayali en el noreste de la Amazonía peruana registraron 56 especies de palmas en sólo 35 transectos, que abarcaron los mismos cuatro hábitats (Balslev et al., 2010). Además, Paniagua et al. (2014) describen 29 especies de palmeras y sus usos en la región San Martín, por lo que, se observan más especies de palmeras en otras regiones. Sin embargo, cabe resaltar que el área de del bosque de palmeras de Molinopampa es relativamente pequeña, por lo que considerando la diversidad de climas y altitudes que encontramos en la región Amazonas se puede decir que este número podría ser superior.

Como ya hemos visto, el bosque de palmeras, está compuesto por cuatro especies del género Ceroxylon. Se reportaron un total de 6638 individuos reconocidos en los 16 transectos en total, como se observa en la Figura 3, la especie más abúndate fue $C$. quindiuense 


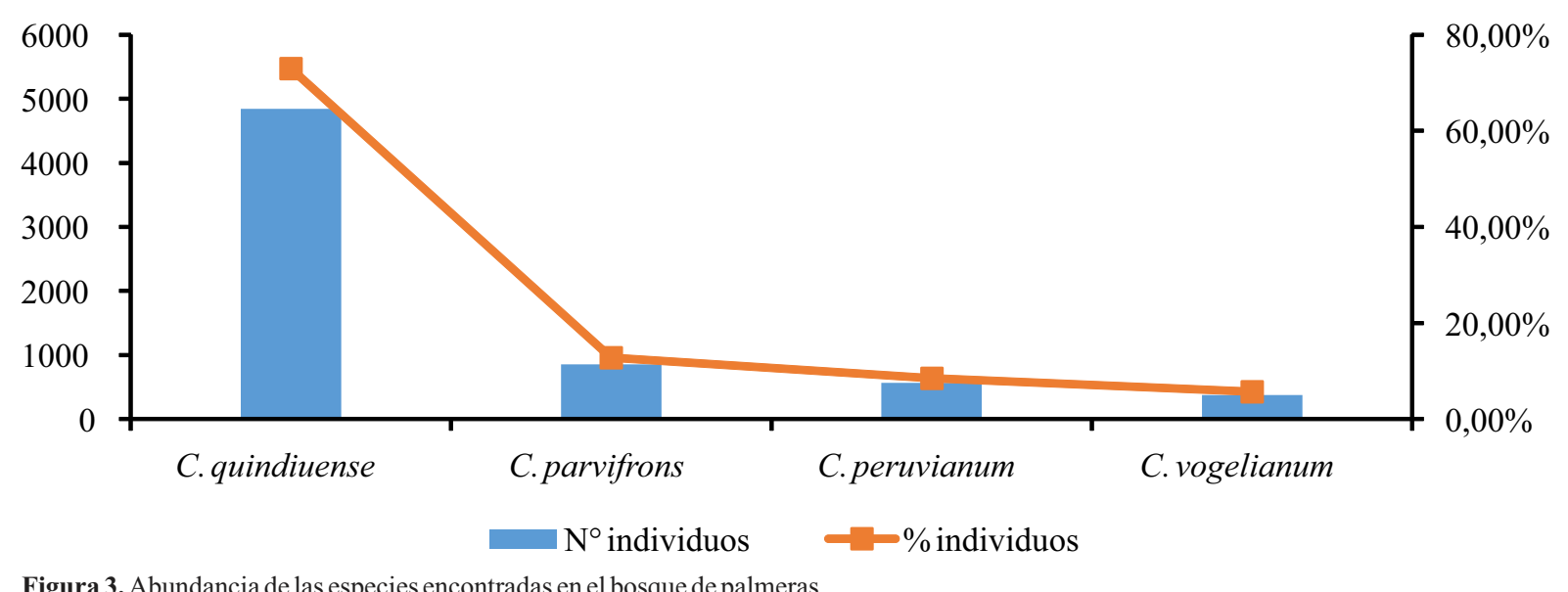

Figura 3. Abundancia de las especies encontradas en el bosque de palmeras

con 4864 individuos lo que representa un $73 \%$ de la población reportada, siendo la dominante en el bosque de palmeras, algo normal dada su distribución en el Norte y Centro de los Andes ya que es la especie de palmeras de cera más representativa de esta zona andina (Sanín y Galeano, 2011). Por otro lado la especie menos abundante es C. vogelianum con 378 individuos, representando tan solo el 5,69 \% de la población, siendo una especie típica acompañante en los diferentes bosques de palmeras del Norte y Centro de los Andes (Sanín et al., 2016).

La baja distribución y por tanto, la baja abundancia de las especies de $C$. peruvianum y $C$. vogelianum se puede atribuir a los efectos generados por la agricultura y la ganadería que se desarrolla en la zona, pues estas reducen la capacidad de propagación natural, especialmente en aquellas áreas donde se desarrolla pastoreo de ganado (Haddad et al., 2015).

En cuanto a la frecuencia, la especie con mayor distribución, basada en su frecuencia, es C. quindiuense ya que estuvo presente en todos los puntos de muestreo (transectos) demostrando su amplia distribución y coincidiendo que es la más abundante, por otro lado las otras especies solo fueron encontradas en un punto de muestreo lo que indica que su distribución es reducida y que no son muy representativas en el ecosistema (Tabla 1).

Además, las diferentes especies fueron encontradas en alturas que van desde los 2272 m.s.n.m., donde se encontró a C. peruvianum, hasta los $2758 \mathrm{msnm}$, como punto más bajo, donde encontramos C. vogelianum.
Tabla 1. Frecuencia de especies según $n^{\circ}$ de transectos evaluados.

\begin{tabular}{ccc}
\hline ESPECIE & $\begin{array}{c}\text { Frecuencia } \\
\text { Absoluta }\end{array}$ & $\begin{array}{c}\text { Frecuencia } \\
\text { Relativa }\end{array}$ \\
\hline C. quindiuense & 16 & $100,00 \%$ \\
C.peruvianum & 1 & $6,25 \%$ \\
C. vogelianum & 1 & $6,25 \%$ \\
C.parvifrons & 1 & $6,25 \%$ \\
\hline
\end{tabular}

Esto es normal, puesto que el género Ceroxylon se distribuye sobre todo en la zona andina, siendo los bosques de palmeros a mayor altitud en el mundo (Pintaud et al., 2008). Dentro del área de estudio, la especie Ceroxylon quindiuense se encontró en los anexos de San José, Puma Hermana, Santa Rosa y Ocol, siendo la que posee una distribución más amplia, por otro lado la especie Ceroxylon peruvianum se limita al anexo de San José, la especie Ceroxylon parvifrons se en el anexo de Puma hermana y la especie Ceroxylon vogelianum únicamente se observó en el anexo San José.

La especie Ceroxylon quindiuense, no es la más abundante solo porque domine totalmente los hábitats en los que se encuentran, si no también, porque parece que tienen una alta adaptabilidad a diferentes ambientes, pudiendo crecer en todos los hábitats con pequeñas variaciones de densidad. (Ruokolainen and Vormisto 2000).Además, los especímenes de esta especie tienden a ser altos y generalistas del hábitat (Gómez-Zapata y Salazar-Yepes, 2017).

Por otro lado, comprobó su diversidad a través del cálculo del índice de Shanon-Weiner, obteniéndose un valor de 0,87 , lo que demuestra la reducida diversidad 
Tabla 2. Índice de diversidad de Shanon-Weiner para las palmeras del genero Ceroxylon en el Bosque de Molinopampa

\begin{tabular}{cccc}
\hline Especie & Abundancia & Frecuencia & Diversidad(H') \\
\hline Ceroxylon quindiuense & 4846 & 0,7300 & $-0,230$ \\
Ceroxylonparvifrons & 851 & 0,1282 & $-0,264$ \\
Ceroxylonperuvianum & 563 & 0,0848 & $-0,209$ \\
Ceroxylonvogelianum & 378 & 0,0569 & $-0,163$ \\
\hline Total & $\mathbf{6 6 3 8}$ & $\mathbf{1}$ & $\mathbf{0 , 8 6 6}$ \\
\hline
\end{tabular}

de especies, sin embargo muestra un a comunidad equitativa, algo muy común en los bosques de palmeras de cera del Norte de los Andes, donde la diversidad suele ser media a baja (Diaz-Puerto y Supelano-Moreno 2016) (Tabla 2). Mediante la aplicación de los índices de diversidad y el valor de importancia se reconoció la relevancia del género Ceroxylon y sus especies.

\section{CONCLUSIONES}

Se encuentra una baja diversidad de especies del genero Ceroxylon, solo se reportan cuatro especies que además se distribuyen en un solo hábitat, siendo la más representativa $C$. quidiuense, presente en todos los transectos y en todas las comunidades.

Se explica la baja distribución por las actividades agrícolas y ganaderas que se ejecutan en el hábitat de las palmeras estudiadas lo que genera un impacto negativo tanto en su diversidad como en su distribución.

\section{REFERENCIAS BIBLIOGRÁFICAS}

Baker, W. J., y J. Dransfield. 2016. "Beyond Genera Palmarum : Progress and Prospects in Palm Systematics.” Botanical Journal of the Linnean Society 182:207-33. doi:10.1111/boj.12401.

Balslev, H., H. Navarrete, N. Paniagua-Zambrana, D. Pedersen, W. Eiserhardt, y T. Kristiansen. 2010. "El Uso de Transectos Para El Estudio de Comunidades de Palmas." Ecología En Bolivia 45 (3): 8-22.

Clark, A. T., M. Detto, H. C. Muller-Landau, S. A. Schnitzer, S. J. Wright, R. Condit, y S. P. Hubbell. 2017. "Functional Traits of Tropical Trees and Lianas Explain Spatial Structure across Multiple Scales.” Journal of Ecology 106 (2): 795-806. doi:10.1111/13652745.12804 .

Clark, A. T., C. Lehman, y D. Tilman. 2018. "Identifying Mechanisms That Structure Ecological Communities by Snapping Model Parameters to Empirically Observed Tradeoffs.” Ecology Letters 21:494-505. doi:10.1111/ele.12910.

Diaz-Puerto, Z., y S. A. Supelano-Moreno. 2016. “Análisis de La Composición Florística En La Reserva Palma de Cera, La Vega (Cundinamarca, Colombia)." Boletín Semillas Ambientales 10 (2): 18-24.

Flores, M., P. Zevallos, R. Baldoceda, y Y. Flores. 2017. “Caracterización Ecológica de Los Bosques de Palmeras Del Centro de Investigación y Capacitación Forestal (CICFOR) Macuya-Huánuco." Mentor Forestal 1: 35-45.

Galeano, G., M. J. Sanín, K. Mejía, J. Pintaud, y B. Millán. 2008. "Novelties in the Genus Ceroxylon (Arecaceae) from Peru, with Description of a New Species." Revista Peruana de Biología 15 (1): 65-72.

Gómez-Zapata, P. A., y M. Salazar-Yepes. 2017. “Camarotella Colombiana Sp. Nov. (Phyllachoraceae) Sobre Ceroxylon Quindiuense (Arecaceae) En Colombia." Revista Mexicana de Biodiversidad 88: $275-79$. doi:10.1016/j.rmb.2017.03.006.

Haddad, N. M., L. A. Brudvig, J. Clobert, K. F. Davies, A. Gonzalez, R. D. Holt, T. E. Lovejoy, et al., 2015. "Habitat Fragmentation and Its Lasting Impact on Earth's Ecosystems." Sci Adv 1: 1-9. doi:10.1126/sciadv.1500052.

Henderson, A., G. Galeano, y R. Bernal. 1995. Field 
Guide to the Palms Of the Americas. New Jersey (EEUU): Princeton University Press.

Hubbell, S. P., y R. B. Foster. 1986. “Biology, Chance and History and the Structure of Tropical Rain Forest Tree Communities." En Community Ecology, Harper and Row Diamond, J. M. y T. J. Case (eds). New York (EEUU): Academic Press. doi:10.4236/gep.2016.47014.

Kahn, F., y F. Moussa. 1994. Las Palmeras Del Perú. Lima (Perú): IFEA.

Manturano, G. D. 2007. Contribución Al Conocimiento Silvicultural de Las Especies Forestales Establecidas Con Fines de Producción Maderera En La Provincia de Satipo. Tesis de Maestría. Univerisdad Nacional Agraria La Molina. Lima (Perú).

Mas, J. F., R. Lemoine-Rodríguez, R. González, J. López-Sánchez, A. Piña-Garduño, y E. Herrera-Flores. 2017. "Evaluación de Las Tasas de Deforestación En Michoacán a Escala Detallada Mediante Un Método Híbrido de Clasificación de Imágenes SPOT." Madera y Bos$q u$ es 23 ( 2 ): $119-31$. doi:10.21829/myb.2017.2321472.

Oliva, M., J. L. Maicelo, C. Torres-Guzmán, y W. Bardales. 2017. "Propiedades Fisicoquímicas Del Suelo En Diferentes Estadios de La Agricultura Migratoria En El Área de Conservación Privada 'Palmeras de Ocol', Distrito de Molinopampa, Provincia de Chachapoyas (Departamento de Amazonas)." Revista de Investigación de Agroproducción Sustentable 1 (1): 9-21.

Paniagua, N., R. Bussmann, C. Vega, C. Téllez, y M. J. Macía. 2014. "Nuestro Conocimiento y Uso de Las Palmeras: Una Herencia Para Nuestros Hijos. Comunidades Llaquash, San Martín, Perú." Ethnobotany Research and Applications 13 (2): 1-105.

Pintaud, J., G. Galeano, H. Balslev, R. Bernal, F. Borchsenius, E. Ferreira, J. Granville, et al., 2008. "Las Palmeras de América Del Sur:
Diversidad, Distribución e Historia Evolutiva." Rev. Peru. Biol. 15 (1): 7-29. doi:10.15381/rpb.v15i3.2662.

Ruokolainen, K., y J. Vormisto. 2000. “The Most Widespread Amazonian Palms Tend to Be Tall and Habitat Generalists." Basic and Applied Ecology 1 (2): 97-108. doi:10.1078/1439-179100020 .

Sanín, M. J., y G. Galeano. 2011. "A Revision of the Andean Wax Palms, Ceroxylon (Arecaceae)." $P h y$ t o t a $x$ a $34: \quad 1-64$. doi:10.11646/phytotaxa.34.1.1.

Sanín, M. J., W. D. Kissling, C. D. Bacon, F. Borchsenius, G. Galeano, J. C. Svenning, J. Olivera, R. Ramírez, P. Trénel, y J. Pintaud. 2016. "The Neogene Rise of the Tropical Andes Facilitated Diversification of Wax Palms (Ceroxylon: Arecaceae) through Geographical Colonization and Climatic Niche Separation." Botanical Journal of the Linnean Society 182: 303-17. doi:10.1111/boj.12419.

Santa-Cruz, Lázaro. 2011. Flora de Espermatofitas Del Distrito de Pulán, Santa CruzCajamarca. Tesis de Maestría. Universidad Nacional Mayor de San Marcos. Lima (Perú).

Wright, S. J., y H. C. Duber. 2001. "Poachers and Forest Fragmentation Alter Seed Dispersal, Seed Survival, and Seedling Recruitment in the Palm Attalea Butyraceae, with Implications for Tropical Tree Diversity." Biotropica 33 (4): 583-95. doi:10.1111/j.17447429.2001.tb00217.x. 